\title{
PREDICTING THE PROPERTIES OF PURE BREEDING LINES EXTRACTABLE FROM A CROSS IN THE PRESENCE OF LINKAGE
}

\author{
J. L. JINKS and H. S. POONI
}

Department of Genetics, University of Birmingham, Birmingham B15 2TT, England

Received 23.vi.82

\section{SUMMARY}

The quantitative consequences of using estimates of additive genetic components of variation from the early generations of a cross to predict the properties of the pure breeding lines extractable from it when there is a linkage disequilibrium are investigated theoretically and by examples drawn from the cross of varieties 1 and 5 of Nicotiana rustica. In the presence of a linkage disequilibrium very good predictions can be obtained by combining estimates of the rank 1 and 2 forms of the additive genetic component of variation which can be estimated from $F_{3}$ and $S_{3}$ families of a cross. These predictions can be marginally improved by also estimating the rank 3 form but this requires the addition of $F_{4}$ families.

\section{INTRODUCTION}

THE methods currently used to predict the properties of the pure breeding lines extractable from a cross by pedigree breeding, single seed descent and haploidy assume no linkage although they can detect its presence and direction and allow for its effects qualitatively (Jinks and Perkins, 1972; Jinks and Pooni, 1976, 1980; Pooni and Jinks, 1978, 1979). A linkage disequilibrium after summing over all loci, although not linkage per se, will lead to an underestimate or overestimate of the expected variation among the extractable pure breeding lines for an excess of repulsion and coupling linkages, respectively. In this paper we specify in more detail than previously given the effects of a linkage disequilibrium on the predicted and observed properties of the pure breeding lines and show how these effects may be allowed for to improve prediction in its presence.

\section{THEORY}

The total additive genetic variance in the $n$th generation of inbreeding by self-pollination $\left({ }_{D} V_{F n}\right)$ from a cross between a pair of pure breeding lines is given by the summation $\sum_{r=1}^{n-1}\left(\frac{1}{2}\right)^{r} D_{r}$ where $r$ is rank (Mather and Jinks, 1971). Each of the $r=1$ to $n-1$ ranks in the $n$th generation has the general expectation

$$
D_{r}=\sum_{i=1}^{k} d_{i-R}^{2+C} \sum_{i=1}^{k-1} \sum_{j=i+1}^{k} 2\left(1-2 p_{i j}\right)^{r} d_{i} d_{j}
$$

where the linkage component, $2\left(1-2 p_{i j}\right)^{r} d_{i} d_{j}$ is added for each pair of genes linked in coupling $(C)$ and subtracted for each pair linked in repulsion $(R)$. For example, in the $\mathrm{F}_{4}$ the total additive genetic variance $\left({ }_{D} V_{F 4}\right)$ is

$$
\begin{aligned}
& \frac{1}{2} D_{1}+\frac{1}{4} D_{2}+\frac{1}{8} D_{3} \\
& 265
\end{aligned}
$$


where

$$
\begin{aligned}
& D_{1}=\sum_{i=1}^{k} d_{i-R}^{2+C} \sum_{i=1}^{k-1} \sum_{j=i+1}^{k} 2\left(1-2 p_{i j}\right) d_{i} d_{j} \\
& D_{2}=\sum_{i=1}^{k} d_{i-R}^{2+C} \sum_{i=1}^{k-1} \sum_{j=i+1}^{k} 2\left(1-2 p_{i j}\right)^{2} d_{i} d_{j}
\end{aligned}
$$

and

$$
D_{3}=\sum_{i=1}^{k} d_{i-R}^{2+C} \sum_{i=1}^{k-1} \sum_{j=i+1}^{k} 2\left(1-2 p_{i j}\right)^{3} d_{i} d_{j}
$$

When $n$ is large (symbolised as $n=\infty$ ) that is, the inbred families extracted from the cross are pure breeding, the total additive genetic variance among them $\left({ }_{D} V_{F \infty}\right)$ is found as the sum from $r=1$ to $\infty$. The sum of this series

$$
{ }_{D} V_{F \infty}=\frac{1}{2} D_{1}+\frac{1}{4} D_{2}+\frac{1}{8} D_{3}+\frac{1}{16} D_{4}+\frac{1}{32} D_{5}+\cdots+\left(\frac{1}{2}\right)^{\infty} D_{\infty}
$$

reduces to

$$
\sum d_{i-R}^{2+C} \sum_{i=1}^{k-1} \sum_{j=i+1}^{k} \frac{2\left(1-2 p_{i j}\right) d_{i} d_{j}}{1+2 p_{i j}}
$$

Because the coefficients of $D_{r}$ follow the geometric series $\left(\frac{1}{2}\right)^{r}$, successive terms make a rapidly decreasing contribution to the total. Furthermore, because the coefficients of the linkage components of $D$, also follow the geometric series $\pm \sum_{i=1}^{k-1} \sum_{i=i+1}^{k} 2\left(1-2 p_{i j}\right)^{r} d_{i} d_{j}$, the successive $D_{r}$ 's in the series show rapidly falling differences. Hence $\left|D_{1}-D_{2}\right|>\left|D_{2}-D_{3}\right|>$ $\left|D_{3}-D_{4}\right|>\left|D_{4}-D_{5}\right|>\cdots$. Not surprisingly, therefore, $\frac{1}{2} D_{1}+\frac{1}{4} D_{2}+\frac{1}{4} D_{3}$ which assumes that $D_{3}=D_{4}=D_{5} \ldots$, from which it follows that $\frac{1}{8} D_{3}=$ $\frac{1}{16} D_{4}+\frac{1}{32} D_{5}+\cdots$ is a very close approximation to ${ }_{D} V_{F \infty}$ for all values of $p$, the recombination frequency. Indeed $\frac{1}{2} D_{1}+\frac{1}{2} D_{2}$, which assumes that $D_{2}=D_{3}=D_{4}=D_{5}=\cdots$, from which it follows that $\frac{1}{4} D_{2}=\frac{1}{8} D_{3}+\frac{1}{16} D_{4}+$ $\frac{1}{32} D_{5}+\cdots$ is an acceptable approximation in practice to ${ }_{D} V_{F \infty}$.

The closeness of these approximations to the true value of ${ }_{D} V_{F \infty}$ is illustrated in table 1. The upper half of the table contains the values of $D_{r}$ for $r=1$ to 5 for a pair of genes linked in coupling and in repulsion each with an additive effect $d=1$ and recombination frequencies of $p=0 \cdot 1$, 0.25 and 0.4 . The lower half of the table contains the true values of ${ }_{D} V_{F \infty}$ corresponding with these parameter values and approximate predictions of ${ }_{D} V_{F \infty}$ based upon the first few terms in the summation series, ${ }_{D} V_{F \infty}=$ $\sum_{r=1}^{\infty}\left(\frac{1}{2}\right)^{r} D_{r}$.

Reference to table 1 shows that irrespective of the linkage phase or of the recombination frequency, very close approximations to the total genetic variance among the means of the pure breeding families of the $F_{\infty}$ generation $\left.{ }_{D} V_{F \infty}\right)$ can be obtained without going beyond $D_{3}$ or even $D_{2}$ in the summation series. It follows, therefore, that any breeding programme that will yield estimates of $D_{1}, D_{2}$ and possibly $D_{3}$ will allow acceptable predictions of the distribution of the means of the pure breeding families extractable from a cross. 
TABLE 1

The values of $\mathrm{D}_{\mathrm{r}}$ for $\mathrm{r}=1$ to 5 for $\mathrm{p}=0.1,0.25$ and 0.4 , the true values of $\mathrm{D}_{\mathrm{F} \infty}$ and approximate predictions based upon the first few terms in the summation series ${ }_{\mathrm{D}} \mathrm{V}_{\mathrm{F} \infty}=$ $\sum_{r=1}^{\infty}\left(\frac{1}{2}\right)^{r} D_{r}$

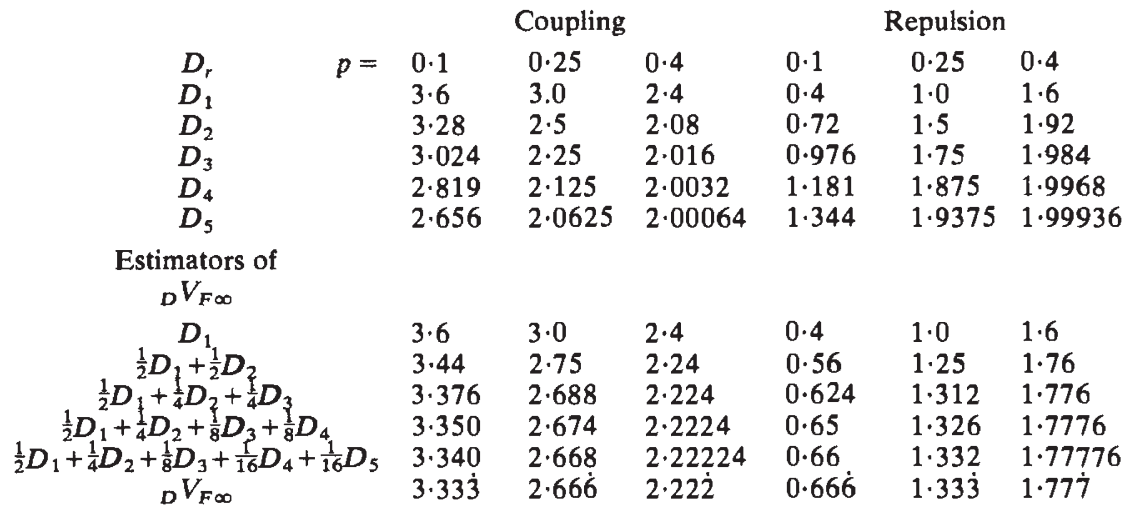

\section{EXPERIMENTAL DESIGNS}

Estimation of the additive genetic components of variance of different rank, that is $D_{1}, D_{2}$ and $D_{3}$ is described by Mather and Jinks $(1971,1982)$. The usual approach is a pedigree inbreeding design using self-pollination (Mather, 1949, Virk et al., 1981) or sib-mating (Cooke and Mather, 1962). Unless the non-additive genetic sources of variation are non-significant the inbreeding series must be taken as far as the fourth generation $\left(F_{4}\right)$ to obtain estimates of $D_{1}$ and $D_{2}$ and to the fifth generation to estimate $D_{3}$ also. Because of the relatively small contribution that non-additive sources such as dominance make to the variation following inbreeding they can be non-significant even when the dominance ratio is high. Hence it will often be correct to assume that the non-additive sources of variation make no significant contributions so that $D_{1}$ and $D_{2}$ can be estimated by the $\mathrm{F}_{3}$ or $S_{3}$ and $D_{3}$ also by the $F_{4}$ and $S_{4}$.

Other designs will estimate $D_{1}$, the best estimates being provided by an $F_{2}$ triple test cross (Kearsey and Jinks, 1968) but $D_{2}$ cannot be obtained earlier than the $F_{3}$ or $S_{3}$. By combining the two inbreeding series, selfing and sib mating, $D_{1}$ and $D_{2}$ can be estimated even in the presence of a significant dominance component of variation from the $F_{3}$ and $S_{3}$. Thus from a random sample of $F_{2}$ individuals we can simultaneously produce $F_{3}$ progeny families by selfing and $S_{3}$ progeny families by sib mating pairs at random. From an appropriately randomised and replicated experiment the $F_{3}$ and $S_{3}$ progeny families will yield estimates of between and within family components of variance with the expectations (Mather and Jinks, 1982):

Between family means $\quad V_{1 F 3}=\frac{1}{2} D_{1}+\frac{1}{16} H_{1}, \quad V_{1 S 3}=\frac{1}{4} D_{1}+\frac{1}{16} H_{1}$

Within families $\quad V_{2 F 3}=\frac{1}{4} D_{2}+\frac{1}{8} H_{2}+E_{w}, \quad V_{2 S 3}=\frac{1}{4} D_{2}+\frac{3}{16} H_{2}+E_{w}$.

Estimates of $E_{w}$, the within family environmental component of variation, can be obtained in the usual way by including the parental, $P_{1}$ and $P_{2}$ and their $F_{1}$ families in the experiment. A perfect fit solution is then 
possible in which

$$
\begin{aligned}
& D_{1}=4\left(V_{1 F 3}-V_{1 S 3}\right) \\
& D_{2}=12 V_{2 F 3}-8 V_{2 S 3}-4 E_{w} \\
& H_{1}=32 V_{1 S 3}-16 V_{1 F 3} \\
& H_{2}=16\left(V_{2 S 3}-V_{2 F 3}\right) .
\end{aligned}
$$

We can now predict ${ }_{D} V_{F \infty}$ as

$$
\frac{1}{2} D_{1}+\frac{1}{2} D_{2}=2 V_{1 F 3}+6 V_{2 F 3}-2 V_{1 S 3}-4 V_{2 S 3}-2 E_{w} \text {. }
$$

\section{EXAMPLES}

An example of the estimation of $D_{1}, D_{2}$ and $D_{3}$ from the $F_{3}$ and $F_{4}$ families of a pedigree selfing series is provided by Virk et al. (1981) and Ingram and Jinks (1982). The cross was between varieties 1 and 5 of Nicotiana rustica and two characters, flowering time and plant height at flowering time showed a significant linkage disequilibrium attributable to an excess of the repulsion phase. Because there was no significant nonadditive variation (section 3 ) $D_{1}, D_{2}$ and $D_{3}$ could be estimated by iterative weighted least squares procedures. For both characters $D_{1}$ was significantly smaller than $D_{2}$ and $D_{3}$ but $D_{2}$ and $D_{3}$ did not differ significantly. We can, therefore, predict ${ }_{D} V_{F \infty}$ as $\frac{1}{2} D_{1}+\frac{1}{2} D_{2}=\frac{1}{2} D_{1}+\frac{1}{4} D_{2}+\frac{1}{4} D_{3}$ (table 1$)$.

The estimates of $D_{1}, D_{2}=D_{3}$, the predictions of ${ }_{D} V_{F \infty}$ based upon them and two direct estimates of it obtained from a random sample of $82 \mathrm{~F}_{15}$ families produced by single seed descent and a random sample of $188 \mathrm{~F}_{6}$ families produced by pedigree inbreeding by self-pollination are presented in table 2 . The predictions, which allow for the linkage disequilibrium, are closer to the observed additive genetic variances in the $F_{6}$ and $F_{15}$ than $F_{2}$ triple test cross estimates of 29.9 for FT and 193.6 for HFT obtained from the same experiment, which do not. The latter estimates because of the repulsion linkages are, as expected, smaller.

TABLE 2

Estimates of the rank 1, 2 and 3 additive genetic component of variation (D) and the predicted and observed additive genetic component of variation $\left(\mathrm{D}_{\mathrm{F}}\right)$ among highly inbred families produced by single seed descent $\left(\mathrm{F}_{15}\right)$ and pedigree inbreeding $\left(\mathrm{F}_{6}\right)$

\begin{tabular}{lccccc} 
& \multicolumn{2}{c}{ Estimates } & Predicted ${ }_{D} V_{F \infty}$ & \multicolumn{2}{c}{ Observed ${ }_{D} V_{F \infty}$} \\
\multicolumn{1}{c}{ Character } & $D_{1}$ & $D_{2}=D_{3}$ & $\frac{1}{2} D_{1}+\frac{1}{4} D_{2}+\frac{1}{4} D_{3}$ & $F_{6}$ & $F_{15}$ \\
Flowering time & 21.0 & 67.9 & 44.45 & 39.4 & 38.4 \\
Height at & 92.5 & 384.9 & 238.7 & 271.0 & 275.2
\end{tabular}

\section{Discussion}

In the presence of a linkage disequilibrium the genetical variation in the population of pure breeding lines extractable from a cross by selfpollination is specifiable as the sum of a geometric series in which the terms are in ascending order the rank 1 to $\infty$ forms of the additive genetic component of variation $D$. A very good approximation, however, can be 
obtained from the first three rank forms $D_{1}, D_{2}$ and $D_{3}$ because $D_{1}$ and $D_{2}$ make by far the largest contributions to the sum of the series and $D_{3}$ can be substituted for $D_{4}$ to $D_{\infty}$ because they are unlikely to differ significantly in magnitude (table 1 ). If as is often the case in a selfing series, the non-additive sources of variation such as dominance are making a non-significant contribution, $D_{1}, D_{2}$ and $D_{3}$ can be estimated from the $F_{3}$ and $\mathrm{F}_{4}$ families. If, however, there is a significant dominance contribution $F_{3}$ and $F_{4}$ families or $F_{3}$ and $S_{3}$ families are required to estimate $D_{1}$ and $D_{2}$.

Most of these theoretical points are confirmed by flowering time and plant height at flowering time in the cross of varieties 1 and 5 of Nicotiana rustica (table 2). Although these two characters display dominance (Pooni et al., 1978), in the $F_{3}$ and $F_{4}$ generations its contribution to the variation is too small because of the low level of heterozygosity to be significant, hence $D_{1}, D_{2}$ and $D_{3}$ can be estimated. But whereas, because of the excess of repulsion linkages, $D_{1}$ is significantly smaller than $D_{2}$ and $D_{3}, D_{2}$ and $D_{3}$ do not differ significantly. The estimate of $D_{2}=D_{3}$ can, therefore, be substituted for $D_{4}$ to $D_{\infty}$ in predicting the expected additive genetic variance among the pure breeding lines extracted from the cross. This prediction is more accurate than the best estimate obtained from an $F_{2}$ triple test cross which makes no allowance for linkage. Nevertheless, it should be noted that while there is this improvement in accuracy in the $N$. rustica examples the $F_{2}$ triple test cross prediction is close enough for all practical purposes.

Where pure breeding lines are extracted by dihaploidy as opposed to single seed descent or pedigree inbreeding the extraction usually takes place from the earliest generations of a cross. There can be no contribution of recombination in generations subsequent to the one chosen for extraction to the genetical variation among the pure breeding lines. Hence $D_{1}, D_{2}$ and occasionally $D_{3}$ are the only contributors to this variation (Jinks and Pooni, 1981) and the only rank forms of $D$ that need to be estimated in order to predict its magnitude.

\section{REFERENCES}

COOKE, P., AND MATHER, K. 1962. Estimating the components of continuous variation. II Genetical. Heredity, 17, 211-236.

INGRAM, N. R., AND JINKS, J. L. 1982. Analysis of induced quantitative variation. II. The recombinant inbred lines. Heredity, 48, 79-83.

JINKS, J. L., AND PERKINS, J. M. 1972. Predicting the range of inbred lines. Heredity, 28, $399-403$.

JINKS, J. L., AND POONI, H. S. 1976. Predicting the properties of recombinant inbred lines derived by single seed descent. Heredity, 36, 253-266.

JINKS, J. L., AND POONI, H. S. 1980. Comparing predictions of mean performance and environmental sensitivity of recombinant inbred lines based upon $F_{3}$ and triple test cross families. Heredity, 45, 305-312.

JINKS, J. L.. AND POONI, H. S. 1981. Properties of pure breeding lines produced by dihaploidy, single seed descent and pedigree breeding. Heredity, 46, 391-395.

KEARSEY, M. J., AND JINKS, J. L. 1968. A general method of detecting additive, dominance and epistatic variation for a metrical trait. I. Theory. Heredity, 23, 403-409.

MATHER, K. 1949. Biometrical Genetics (First Edition). Methuen, London.

MATHER, K., AND JINKS, J. L. 1971. Biometrical Genetics (Second Edition) Chapman and Hall, London.

MATHER, K., AND JINKS, J. L. 1982. Biometrical Genetics (Third Edition) Chapman and Hall, London. 
POONI, H. S., AND JINKS, J. L. 1978. Predicting the properties of recombinant inbred lines derived by single seed descent for two or more characters simultaneously. Heredity, 40, 349-361.

POONI, H. S., AND JINKS, J. L. 1979. Sources and biases of predictors of the properties of recombinant inbreds produced by single seed descent. Heredity, 42, 41-48.

POONI, H. S., JINKS, J. L., AND JAYASEKARA, N. E. M. 1978. An investigation of gene action and genotype $\times$ environment interaction in two crosses of Nictoniana rustica by triple test cross and inbred line analysis. Heredity, 41, 83-92.

VIRK, D. S., POONI, G. S., INGRAM, N. R., AND JINKS, J. L. 1981. Analysis of induced quantitative variation. I. Hierarchical and triple test cross analysis. Heredity, 47, 317-326. 